\title{
Magnetic resonance imaging in the evaluation of cervical foraminal stenosis: comparison of 3D T2 SPACE with sagittal oblique 2D T2 TSE
}

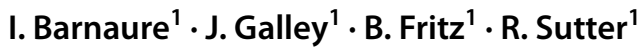 \\ Received: 16 November 2021 / Revised: 30 December 2021 / Accepted: 2 January 2022 / Published online: 10 January 2022 \\ (c) The Author(s) 2022
}

\begin{abstract}
Objective The oblique orientation of the cervical neural foramina challenges the implementation of a short MRI protocol with concurrent excellent visualization of the spine. While sagittal oblique T2-weighted sequences permit good evaluation of the cervical neuroforamina, all segments may not be equally well depicted on a single sequence and conspicuity of foraminal stenosis may be limited. 3D T2-weighted sequences can be reformatted in arbitrary planes, including the sagittal oblique. We set out to compare 3D T2w SPACE sequences with sagittal oblique reformations and sagittal oblique 2D T2w TSE sequences for the evaluation of cervical foraminal visibility and stenosis.

Materials and methods Sixty consecutive patients who underwent MRI of the cervical spine with sagittal oblique 2D T2w TSE and 3D T2w SPACE sequences were included. Image homogeneity of the sequences was evaluated. Imaging sets were assessed for structure visibility and foraminal stenosis by two independent readers. Results of the sequences were compared by Wilcoxon matched-pairs tests. Interreader agreement was evaluated by weighted $\kappa$.

Results Visibility of most structures was rated good to excellent on both sequences (mean visibility scores $\geq 4.5$ of 5 ), though neuroforaminal contents were better seen on sagittal oblique T2w TSE (mean scores 4.1-4.6 vs. 3.1-4.1 on 3D T2w SPACE, $p<0.01$ ). Stenosis grades were comparable between sequences (mean 1.1-2.6 of 4), with slightly higher values for 3D T2w SPACE at some levels (difference $\leq 0.3$ points).
\end{abstract}

Conclusion 3D T2w SPACE is comparable with sagittal oblique 2D T2w TSE in the evaluation of cervical neural foramina.

Keywords MRI $\cdot$ Cervical Spine $\cdot$ Foraminal stenosis

\section{Abbreviation \\ SPACE Sampling perfection with application optimized contrasts using different flip angle evolutions}

\section{Introduction}

Degenerative diseases of the spine are highly prevalent and account for a large proportion of cases examined in daily clinical practice [1]. MRI, a non-invasive and non-irradiating technique with excellent visualization of the spine and spinal cord, has become the method of choice for the imaging evaluation of the spine. While standard examination planes - mostly sagittal and axial-allow for evaluation of both spinal canal contents and neural foramina in the lumbar

\section{Barnaure}

ibarnaure@gmail.com

1 Department of Radiology, Balgrist University Hospital, Forchstrasse 340, 8008 Zurich, Switzerland spine, visualization of the cervical neural foramina and their contents is rendered more difficult by their oblique orientation, with angles of about $45^{\circ}$ to the sagittal and $10-15^{\circ}$ (inferiorly) to the axial plane [2]. This has led to the use of oblique projections for radiographic evaluation [3], oblique reconstructions of CT acquisitions [4], and oblique sagittal MRI sequences (mostly T2w) angled about $45^{\circ}$ [2, 5-7] for a better, in-plane representation of the foramina. Oblique (or "angled") sagittal T2w sequences have been shown to be more accurate in the evaluation of the cervical neural foramina than "conventional" sagittal and axial sequences [2, 6-8], and their use may alter surgical recommendations [9].

No standard imaging protocol has been established for the examination of the cervical spine [10], and protocols vary among institutions. An ideal protocol would allow excellent visualization of the spine, its contents and its surroundings, combined with a fast acquisition time to avoid patient discomfort and motion and provide enough imaging slots in a context of high demand. While sagittal oblique 
T2w sequences have been shown to permit good evaluation of the cervical neural foramina, these sequences are added to the conventional sequences, thus increasing examination time. 3D sequences allow for reformation in arbitrary planes, including perpendicular to the axis of the neural foramina (i.e., sagittal oblique) and adaptable to each level, and offer coverage of the foramina of both sides and the spinal canal in a single sequence at reasonable acquisition times. 3D T2w SPACE sequences have been used for imaging of the knee [11], lumbar [12], and cervical spine [13, 14] and have been evaluated in comparison with conventional $2 \mathrm{D}$ sequences, with a focus on structure visibility and comparison to conventional sagittal and axial sequences in the cervical spine [13-16].

The purpose of this study was to compare sagittal oblique 2D T2w TSE sequences of the spine and 3D T2w SPACE sequences with sagittal oblique reformations for the evaluation of the cervical neural foramina.

\section{Material and methods}

\section{Subjects}

The local ethics committee approved this retrospective study. Sixty consecutive patients who underwent MRI of the cervical spine at our institution were enrolled with the following inclusion criteria: (i) MRI of the cervical spine at our institution including sagittal oblique 2D T2-weighted and sagittal 3D T2-weighted sequences; (ii) referral by the institution's orthopedic surgeons for cervical pain, cervical radiculopathy, or degenerative disease of the spine; and (iii) age $>18$ years.

\section{Imaging parameters}

Examinations were performed on a 1.5 Tesla or a 3 TeslaMR scanner (Avanto-fit and Skyra-fit, respectively, Siemens Healthineers) with a 20-channel head/neck coil. The protocol included sagittal T2w and T1w TSE sequences, an axial T2w TSE sequence, sagittal oblique T2w TSE sequences angled and centered on the neuroforamina of both sides ("sagittal oblique T2w"), and a sagittal 3D T2w sequence (SPACE, Sampling Perfection with Application optimized Contrasts using different flip angle Evolutions). Details of the sequences are listed in Table 1.

\section{Image analysis}

\section{Quantitative analysis}

To compare T2w TSE and 3D T2w SPACE sequences, their image heterogeneity or non-uniformity (NU) was assessed, defined as $N U=\frac{S D R O I}{\text { SIROI }} \times 100$, with SI as the signal intensity and SD as its standard deviation within a region of interest (ROI). Higher non-uniformity values indicate higher signal heterogeneity and potentially higher background noise [17]. Assessed locations were cerebrospinal fluid (CSF) (ROI of $5 \mathrm{~mm}^{2}$ ), fat, bone, and muscle (all with ROIs of $10 \mathrm{~mm}^{2}$ ).

\section{Qualitative analysis}

Two readers (fellowship-trained radiologists with 8 and 6 years of experience, respectively) independently evaluated the images. Sagittal oblique T2-weighted sequences (2D T2w TSE) and sagittal 3D T2-weighted sequences (3D $\mathrm{T} 2 \mathrm{w}$ SPACE) of each patient were presented as separate

Table 1 Imaging parameters

\begin{tabular}{|c|c|c|c|c|}
\hline & \multicolumn{2}{|l|}{$1.5 \mathrm{~T}$} & \multicolumn{2}{|l|}{$3 \mathrm{~T}$} \\
\hline & T2w TSE & T2w SPACE & T2w TSE & T2w SPACE \\
\hline TR (ms) & 3000 & 1500 & 3000 & 1500 \\
\hline $\mathrm{TE}(\mathrm{ms})$ & 83 & 123 & 94 & 129 \\
\hline FA $\left(^{\circ}\right)$ & 150 & 120 & 150 & 100 \\
\hline FOV (mm) & $220 \times 220$ & $240 \times 240$ & $220 \times 220$ & $240 \times 240$ \\
\hline Matrix & $384 \times 288$ & $256 \times 256$ & $320 \times 272$ & $320 \times 320$ \\
\hline Slice thickness (mm) & 3 & 0.94 & 2.5 & 0.94 \\
\hline Gap (mm) & 0.3 & - & 0.25 & - \\
\hline Slices (n) & $11(\times 2)$ & 56 & $12(\times 2)$ & 52 \\
\hline iPAT factor & - & 3 & - & 2 \\
\hline TA (min:s) & $02: 27(\times 2)$ & $04: 28$ & $01: 33(\times 2)$ & 04:31 \\
\hline
\end{tabular}

$T R$ time of repetition, $T E$ echo time, $F A$ flip angle, $F O V$ field of view, $I P A T$ integrated parallel acquisition technique, $T A$ acquisition time. The T2w TSE sequences were acquired in a sagittal oblique plane $\left(45^{\circ}\right)$ centered on the neuroforamina: Two acquisitions were performed in each patient $(\times 2)$, one per side. For the T2w SPACE, only a single acquisition was performed 
anonymized image sets and were evaluated in different sessions, separated by an interval of 2 weeks, using the institution's Picture Archiving and Communication System (PACS) viewer (Merlin, Phoenix-PACS). 2D T2w TSE and 3D T2w SPACE were analyzed in alternating blocks of 10 cases. 3D T2w sequences were reformatted by each reader independently with the PACS system's Multiplanar Reconstruction (MPR) tool to produce sagittal oblique images that could be angled differently for the analysis of each level, only the sagittal oblique view being used for analysis.

\section{Artifacts and structure visibility}

Artifacts (flow, pulsation, motion) were graded on a 4-point scale (1: no artifacts, excellent image quality, no limitations (sharp delineation even of small structures), 2: mild artifacts, image quality acceptable, detail detection possible (subtle blurring, but preserved identifiability of structures), 3: considerable artifacts, image quality limited, detail detection hampered, 4: severe artifacts, image quality not acceptable) and visibility of anatomical structures was graded on a 5-point Likert scale (5: excellent visibility, 4: good visibility, 3 : adequately visible, 2 : barely visible 1 : not visible), both adapted from Meindl et al. [13]. Evaluated structures were neuroforamen (border visibility), neuroforaminal fat, intraforaminal nerve root, intraforaminal vessel, vertebral body, vertebral disk, pedicle, and facet joint.

\section{Stenosis grading}

Foraminal stenosis was graded on a 5-point scale as proposed by Park et al. [18]: 1: grade 0, no stenosis; 2 : grade 1 , mild stenosis with partial ( $<50 \%$ of root circumference) perineural fat obliteration; 3 : grade 2 , moderate stenosis with nearly complete ( $>50 \%$ of root circumference) perineural fat obliteration; and 4: grade 3, severe stenosis with nerve root collapse or morphological changes (examples in Figs. 1, 2, $3,4)$.

Time needed to complete the grading was recorded (including the reformatting process for 3D T2w SPACE). Study data were collected and managed using REDCap electronic data capture tools $[19,20]$.

\section{Statistical analysis}

Differences between 2D T2w TSE and 3D T2w SPACE sequences concerning non-uniformity values and the results of the qualitative analysis (artifacts, structure
Fig. 1 Corresponding a sagittal oblique 2D T2w TSE and b sagittal oblique MPR of 3D T2w SPACE at 1.5 T. Orientation of the images shown on $\mathbf{c}$ for the $2 \mathrm{D}$ and $\mathbf{d}$ for the $3 \mathrm{D}$ sequence. C5/C6 foraminal stenosis (arrow) with obliteration of $<50 \%$ of the perineural fat (grade 1 stenosis), but apparent deformity of the C6 nerve root on $\mathbf{a}$, not present on $\mathbf{b}$. No stenosis at the other depicted levels
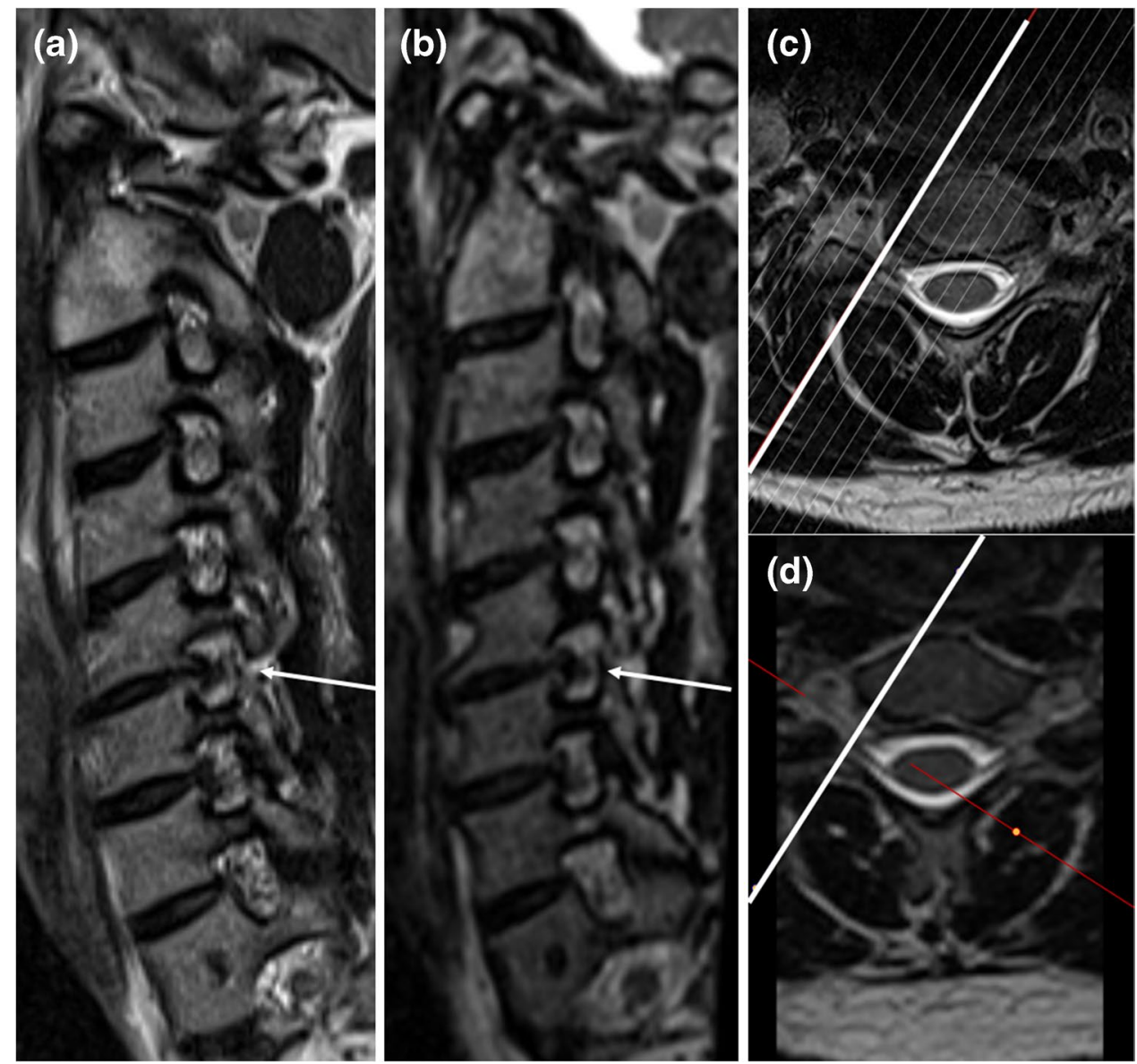
Fig. 2 Corresponding a sagittal oblique 2D T2w TSE and b sagittal oblique MPR of 3D T2w SPACE at $3 \mathrm{~T}$. Severe stenosis of the $\mathrm{C} 6 / \mathrm{C} 7$ foramen (arrow) with total obliteration of the perineural fat and deformity of the $\mathrm{C} 7$ nerve root apparent on both sequences
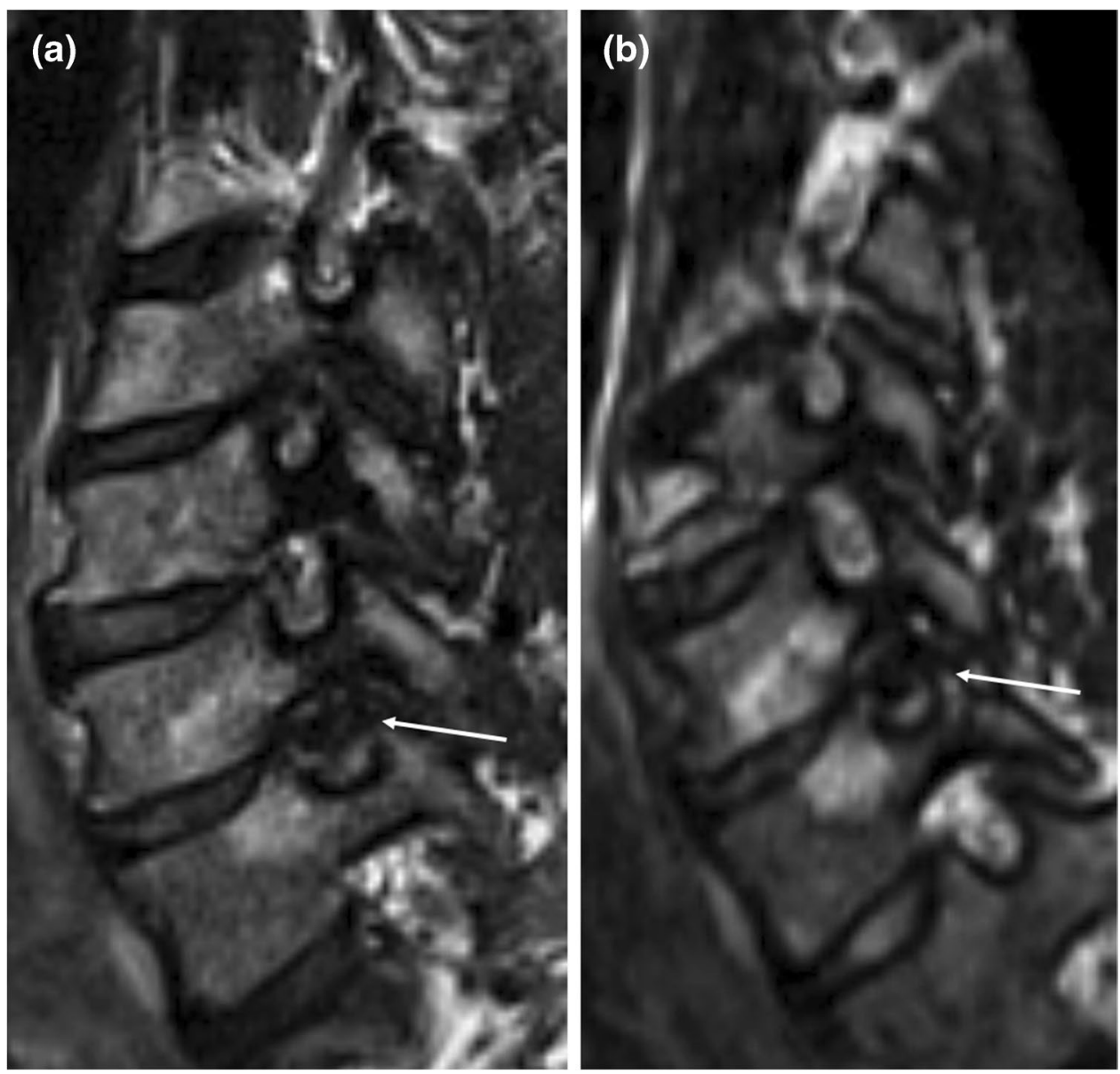

visibility, foraminal stenosis) were tested for statistical significance by use of a Wilcoxon matched-pairs signedranks test (using the mean values of the two readers for the qualitative evaluation). A $p$-value of $<0.05$ was considered statistically significant. Interreader agreement was assessed by means of a weighted $\kappa$ correlation coefficient, interpreted as poor $(\kappa<0)$, slight $(\kappa 0-0.2)$, fair $(\kappa$

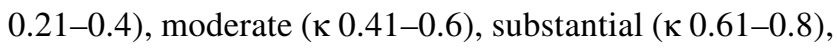
or almost perfect $(\kappa>0.8)$ [21].

Statistical analyses were performed using SPSS version 24.0 (IBM Corp.) and MedCalc version 17.6 (MedCalc Software bvba), the latter for calculation of the weighted kappa.

\section{Results}

The 60 included patients had a mean age of 48 years (standard deviation 14.1 years, range $20-80$ years) and consisted of 31 men (52\%) and 29 women (48\%). Forty patients $(66.7 \%)$ were imaged at $1.5 \mathrm{~T}$ and $20(33.3 \%)$ at $3 \mathrm{~T}$.

\section{Quantitative analysis}

Non-uniformity values of 2D T2w TSE and 3D T2w SPACE sequences at $1.5 \mathrm{~T}$ and $3 \mathrm{~T}$ are presented in Table 2: Significant differences between the sequences were found at $1.5 \mathrm{~T}$, the non-uniformity of CSF being lower for 3D T2w SPACE (4.77 (2.32 SD) vs. $5.72(2.39 \mathrm{SD}), p=0.022)$ and higher for bone (13.22 (3.63 SD) vs. 11.25 (2.74 SD), $p<0.01)$ and muscle (34.2 (9.15 SD) vs. 23.75 (4.38 SD), $p<0.01$ ). Differences between 3D T2w SPACE and 2D T2w TSE were not significant at $3 \mathrm{~T}$ except for muscle, with a higher nonuniformity for the 3D T2w SPACE sequence (31.9 (14.57 $\mathrm{SD})$ vs. $21.11(5.31 \mathrm{SD}), p<0.01)$.

\section{Artifacts and structure visibility}

Artifacts of the investigated sequences were rated as nonexistent to mild (Table 3), without a significant difference between 3D T2w SPACE and 2D T2w TSE when considering all cases (average score of 1.5 for each, $p=0.745$ ), but with higher scores for the 2D T2w TSE sequence at $3 \mathrm{~T}(1.7$ vs. $1.4 p=0.023)$ and for the $3 \mathrm{D} \mathrm{T} 2 \mathrm{w}$ SPACE sequence at $1.5 \mathrm{~T}$ ( 1.6 vs. $1.5, p=0.030)$. 
Fig. 3 Corresponding a sagittal oblique 2D T2w TSE and b sagittal oblique MPR of 3D T2w SPACE at $1.5 \mathrm{~T}$. C4/C5 discoosteophytic foraminal stenosis (arrow) with obliteration of $<50 \%$ of the perineural fat (grade 1 stenosis), but apparent deformity of the C5 nerve root on $\mathbf{a}$, not present on $\mathbf{b}$. No stenosis at the other depicted levels

Fig. 4 Corresponding a sagittal oblique 2D T2w TSE and b sagittal oblique MPR of 3D T2w SPACE at 1.5 T. Severe stenosis of the $\mathrm{C} 6 / \mathrm{C} 7$ foramen (arrow) with total obliteration of the perineural fat and deformity of the $\mathrm{C} 7$ nerve root apparent on both sequences
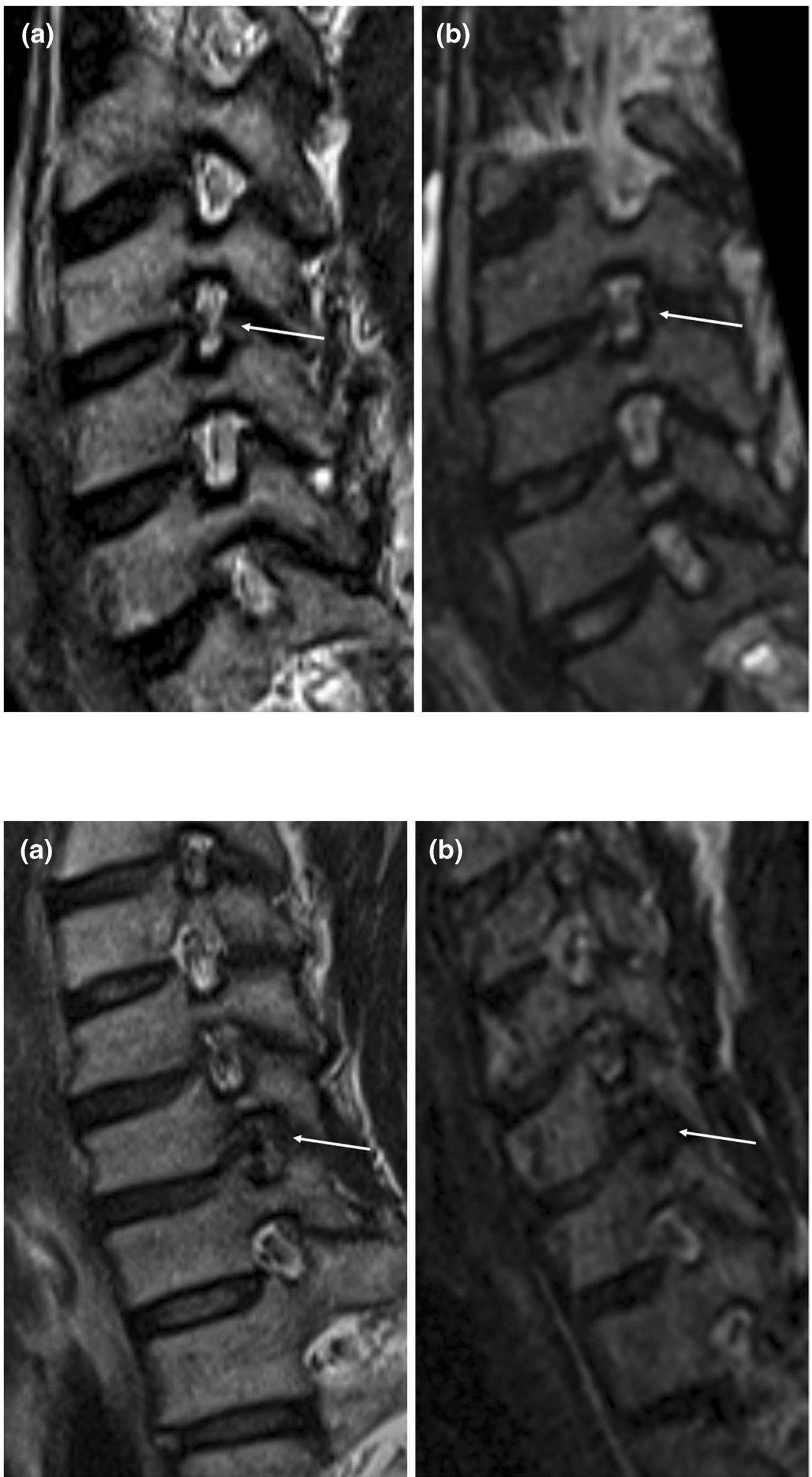
Table 2 Non-uniformity values of the investigated sequences

\begin{tabular}{llllr}
\hline Field strength & Structure & T2w TSE & T2w SPACE & $p$-value \\
\hline Combined & CSF & $6.02(2.45)$ & $5.26(3.2)$ & 0.028 \\
& Fat & $8.39(3.48)$ & $9.31(4.74)$ & 0.245 \\
& Bone & $11.59(2.95)$ & $12.94(4.07)$ & 0.005 \\
$1.5 \mathrm{~T}$ & Muscle & $22.87(4.83)$ & $33.43(11.18)$ & $<0.001$ \\
& CSF & $5.72(2.39)$ & $4.77(2.32)$ & 0.022 \\
& Fat & $7.91(2.83)$ & $9.16(3.95)$ & 0.090 \\
$3 \mathrm{~T}$ & Bone & $11.25(2.74)$ & $13.22(3.63)$ & 0.001 \\
& Muscle & $23.75(4.38)$ & $34.2(9.15)$ & $<0.001$ \\
& CSF & $6.61(2.51)$ & $6.25(4.38)$ & 0.455 \\
& Fat & $9.37(4.43)$ & $9.59(6.14)$ & 0.852 \\
& Bone & $12.29(3.29)$ & $12.39(4.9)$ & 1.000 \\
& Muscle & $21.11(5.31)$ & $31.9(14.57)$ & $<0.001$ \\
\hline
\end{tabular}

Comparison using a Wilcoxon matched-pairs signed-rank test

Table 3 Artifact grading

\begin{tabular}{llll}
\hline Field strength & T2w TSE & T2w SPACE & $p$-value \\
\hline All cases & $1.5(0.34)$ & $1.5(0.33)$ & 0.745 \\
$1.5 \mathrm{~T}$ & $1.5(0.32)$ & $1.6(0.36)$ & 0.030 \\
$3 \mathrm{~T}$ & $1.7(0.34)$ & $1.4(0.24)$ & 0.023 \\
\hline
\end{tabular}

1 no - 4 severe artifacts. Mean scores (and standard deviation) of the two readers. Comparison using a Wilcoxon matched-pairs signedrank test

Visibility scores of the evaluated anatomical structures on 3D T2 $\mathrm{w}$ SPACE and 2D T2w TSE are presented in Table 4. Visibility of most structures was rated as good to excellent on both sequences, with the exception of the foraminal vessel and the nerve root, whose visibility was rated as adequate on 3D T2 $\mathrm{w}$ SPACE at $1.5 \mathrm{~T}$ and $3 \mathrm{~T}$ for the former and at $3 \mathrm{~T}$ for the latter (with good visibility at $1.5 \mathrm{~T}$ ). Significant differences between the two sequences were found for neuroforaminal fat (mean score of 4.6 vs. 4.1), nerve root ( 4.3 vs. 3.7$)$, and foraminal vessel ( 4.1 vs. 3.1$)$, with a better visibility on 2D T2w TSE, as well as facet joint (4.6 vs. 4.7), with a better visibility on 3D T2w SPACE. The highest difference in mean visibility scores between sequences was 1 point for the foraminal vessel ( 3.1 on $3 \mathrm{D}$ T2w SPACE vs. 4.1 on $2 \mathrm{D}$ T $2 \mathrm{w}$ TSE, $p<0.01)$.

\section{Foraminal stenosis grading}

A little over half of the evaluated segments showed a stenosis: $53 \%$ of foramina based on the 2D T2W TSE sequence and $56 \%$ based on the 3D T2w SPACE sequence, with a minority of severe stenoses (Table 5).

Results of foraminal stenosis grading are shown in Table 6. Mean stenosis grades were low, ranging from 1.1 to 2.6. Significant differences between gradings based on 3D T2 $w$ SPACE and 2D T2w TSE were found at five locations: $\mathrm{C} 3 / \mathrm{C} 4$ and $\mathrm{C} 4 / \mathrm{C} 5$ on both sides and $\mathrm{C} 5 / \mathrm{C} 6$ on the left, with higher grades on 3D T2w SPACE (e.g., C3/C4 on the left with scores of 1.7 vs. $2.0, p<0.01$, mean scores of the two readers).

Interreader agreement was moderate to substantial for both 3D T2w SPACE and 2D T2w TSE (Table 7), except for one level with fair agreement on 3D T2w SPACE (C7/ $\mathrm{T} 1$ right), without consistently higher agreement for any of the sequences.

Time to complete foraminal grading was significantly higher for 3D T2w SPACE for both readers (average of 02:08 min vs. 01:23 for 2D T2 TSE, $p<0.01$ ).

\section{Discussion}

The current study demonstrates comparability of 3D T2w SPACE and sagittal oblique 2D T2w TSE sequences in the evaluation of cervical neural foramina. Results of foraminal

Table 4 Visibility scores of anatomical structures

\begin{tabular}{|c|c|c|c|c|c|c|c|c|c|}
\hline & \multicolumn{3}{|c|}{ All cases combined } & \multicolumn{3}{|l|}{$1.5 \mathrm{~T}$} & \multicolumn{3}{|l|}{$3 \mathrm{~T}$} \\
\hline & T2w TSE & T2w SPACE & $p$-value & T2w TSE & T2w SPACE & $p$-value & T2w TSE & T2w SPACE & $p$-value \\
\hline Neuroforamen & $4.5(0.33)$ & $4.5(0.29)$ & 0.821 & $4.6(0.31)$ & $4.5(0.32)$ & 0.228 & $4.3(0.29)$ & $4.4(0.21)$ & 0.101 \\
\hline Neuroforaminal fat & $4.6(0.33)$ & $4.1(0.47)$ & $<0.001$ & $4.7(0.24)$ & $4.2(0.39)$ & $<0.001$ & $4.3(0.36)$ & $3.7(0.41)$ & $<0.001$ \\
\hline Nerve root & $4.3(0.48)$ & $3.7(0.53)$ & $<0.001$ & $4.4(0.36)$ & $3.9(0.49)$ & $<0.001$ & $4.0(0.59)$ & $3.3(0.41)$ & 0.001 \\
\hline Foraminal vessel & $4.1(0.48)$ & $3.1(0.5)$ & $<0.001$ & $4.2(0.38)$ & $3.1(0.52)$ & $<0.001$ & $3.8(0.56)$ & $3.0(0.46)$ & $<0.001$ \\
\hline Vertebral body & $4.6(0.25)$ & $4.5(0.3)$ & 0.383 & $4.7(0.23)$ & $4.6(0.34)$ & 0.138 & $4.5(0.23)$ & $4.5(0.2)$ & 0.377 \\
\hline Disc & $4.6(0.27)$ & $4.5(0.32)$ & 0.437 & $4.7(0.25)$ & $4.5(0.37)$ & 0.139 & $4.4(0.25)$ & $4.5(0.16)$ & 0.187 \\
\hline Pedicle & $4.7(0.27)$ & $4.7(0.31)$ & 0.623 & $4.8(0.22)$ & $4.8(0.34)$ & 0.520 & $4.5(0.28)$ & $4.7(0.25)$ & 0.041 \\
\hline Facet joint & $4.6(0.24)$ & $4.7(0.38)$ & 0.012 & $4.6(0.22)$ & $4.7(0.44)$ & 0.142 & $4.4(0.22)$ & $4.6(0.22)$ & 0.009 \\
\hline
\end{tabular}

1 , not visible - 5, excellent visibility. Mean scores (and standard deviation) of the two readers for each evaluated structure on both sequences. Comparison using a Wilcoxon matched-pairs signed-rank test 
Table 5 Foraminal stenosis prevalence in the cohort according to both sequences

\begin{tabular}{|c|c|c|c|c|c|}
\hline & Stenosis grade & 1 & 2 & 3 & 4 \\
\hline \multirow[t]{2}{*}{ All cases } & 2D T2w TSE & $341(47.4)$ & $224(31.1)$ & 83 (11.5) & $72(10)$ \\
\hline & 3D T2w SPACE & 317 (44) & $225(31.3)$ & $81(11.3)$ & $97(13.5)$ \\
\hline \multirow[t]{2}{*}{$1.5 \mathrm{~T}$} & 2D T2w TSE & $239(49.8)$ & $154(32.1)$ & $49(10.2)$ & 38 (7.9) \\
\hline & $3 \mathrm{D}$ T2w SPACE & 206 (42.9) & $163(34)$ & $53(11.0)$ & $58(12.1)$ \\
\hline \multirow[t]{2}{*}{$3 \mathrm{~T}$} & 2D T2w TSE & $102(42.5)$ & $70(29.2)$ & $34(14.2)$ & 34 (14.2) \\
\hline & 3D T2w SPACE & $111(46.3)$ & $62(25.8)$ & $28(11.7)$ & 39 (16.3) \\
\hline
\end{tabular}

Number of foramina (\% in parentheses) with each stenosis grade (1 (none)-4 (severe)), based on the mean scores of the 2 readers

grading from both sequences were not significantly different for most levels or showed mostly small differences that appeared within clinically acceptable limits.

\section{Quantitative analysis}

Quantitative analysis revealed differences between the sequences mainly at $1.5 \mathrm{~T}$, in favor of $3 \mathrm{D} \mathrm{T} 2 \mathrm{w}$ SPACE concerning CSF non-uniformity values and in favor of T2w TSE concerning bone and muscle non-uniformity values. Non-uniformity values match those found in previous studies $[13,15]$, though the differences between 2 and 3D T2w sequences were not statistically different in a study at $1.5 \mathrm{~T}$ [13], while they were in a $3 \mathrm{~T}$ cohort [15], in favor of T2w TSE for CSF and muscle. Discrepancy of these findings may be due to technical reasons (different machines and sequence parameters between studies) and differing composition of the study cohorts, with younger volunteers in the mentioned studies and a patient cohort including older subjects in the present study. Overall, the differences in image heterogeneity appear acceptable for clinical use of the sequences.

\section{Artifacts and structure visibility}

Neither 3D T2w SPACE nor 2D T2w TSE was limited by artifacts, and there was no difference between the sequences concerning artifact grading. This is in concordance with the previous results [13]. In contrast, other studies that analyzed different artifact types showed significantly less CSF flow artifact on 3D T2w sequences $[14,15]$. As the current study focused on analysis of the neural foramina, we did not examine artifacts mainly concerning the intraspinal compartment such as CSF flow.

Anatomical structures were well visible on both sequences, with slightly better visibility scores for neuroforaminal contents on 2D T2w TSE, especially for foraminal vessels, whereas previous studies showed better delineation of foraminal contents on 3D T2w sequences $[13,14]$ or no statistical significance between sequences [15]. This may be due to differences in technique including utilization of strictly sagittal planes in those studies, as opposed to the sagittal oblique planes dedicated to foraminal analysis in the present study, allowing optimized foraminal visualization on $2 \mathrm{D} T 2 \mathrm{w}$ TSE and potentially blurring the advantages
Table 6 Foraminal stenosis grading for all cases, as well as separated by field strength

\begin{tabular}{|c|c|c|c|c|c|c|c|c|c|}
\hline & \multicolumn{3}{|c|}{ All cases } & \multicolumn{3}{|c|}{$1.5 \mathrm{~T}$} & \multicolumn{3}{|l|}{$3 \mathrm{~T}$} \\
\hline & $2 \mathrm{D}$ & $3 \mathrm{D}$ & $p$-value & $2 \mathrm{D}$ & $3 \mathrm{D}$ & $p$-value & $2 \mathrm{D}$ & $3 \mathrm{D}$ & $p$-value \\
\hline C2/C3 R & 1.2 & 1.2 & 0.438 & 1.1 & 1.2 & 0.084 & 1.3 & 1.3 & 0.317 \\
\hline C2/C3 L & 1.2 & 1.2 & 0.190 & 1.1 & 1.2 & 0.063 & 1.2 & 1.2 & 0.655 \\
\hline $\mathrm{C} 3 / \mathrm{C} 4 \mathrm{R}$ & 1.8 & 1.9 & 0.043 & 1.7 & 1.9 & 0.105 & 1.9 & 2.0 & 0.222 \\
\hline C3/C4 L & 1.7 & 2.0 & 0.000 & 1.6 & 1.9 & 0.000 & 2.0 & 2.1 & 0.366 \\
\hline C4/C5 R & 1.9 & 2.0 & 0.015 & 1.7 & 2.0 & 0.001 & 2.2 & 2.1 & 0.477 \\
\hline C4/C5 L & 2.0 & 2.2 & 0.037 & 1.9 & 2.2 & 0.002 & 2.4 & 2.2 & 0.353 \\
\hline C5/C6 R & 2.4 & 2.5 & 0.107 & 2.4 & 2.6 & 0.020 & 2.6 & 2.5 & 0.642 \\
\hline C5/C6 L & 2.1 & 2.3 & 0.006 & 2.0 & 2.3 & 0.001 & 2.3 & 2.4 & 0.850 \\
\hline C6/C7 R & 2.0 & 2.1 & 0.042 & 2.0 & 2.1 & 0.012 & 2.0 & 2.0 & 1.000 \\
\hline C6/C7 L & 2.0 & 2.0 & 0.949 & 1.9 & 2.0 & 0.295 & 2.2 & 2.0 & 0.070 \\
\hline C7/T1 R & 1.1 & 1.1 & 0.378 & 1.1 & 1.2 & 0.035 & 1.2 & 1.1 & 0.317 \\
\hline $\mathrm{C} 7 / \mathrm{T} 1 \mathrm{~L}$ & 1.1 & 1.1 & 0.317 & 1.1 & 1.2 & 0.046 & 1.1 & 1.1 & 0.000 \\
\hline
\end{tabular}

1, no stenosis - 4, severe stenosis. 2D and 3D sequences compared by a Wilcoxon matched-pairs signedranks test (using the mean scores of the 2 readers). $R$ right, $L$ left 
Table 7 Foraminal stenosis grading for reader 1 and reader 2

\begin{tabular}{|c|c|c|c|c|c|c|}
\hline & \multicolumn{2}{|c|}{ Reader 1} & \multicolumn{2}{|c|}{ Reader 2} & \multicolumn{2}{|c|}{ Interreader $(\kappa)$} \\
\hline & $2 \mathrm{D}$ & $3 \mathrm{D}$ & $2 \mathrm{D}$ & $3 \mathrm{D}$ & $2 \mathrm{D}$ & $3 \mathrm{D}$ \\
\hline C2/C3 R & 1.1 & 1.2 & 1.2 & 1.2 & 0.677 & 0.760 \\
\hline C2/C3 L & 1.1 & 1.2 & 1.2 & 1.2 & 0.560 & 0.554 \\
\hline C3/C4 R & 1.7 & 1.8 & 1.9 & 2.0 & 0.680 & 0.703 \\
\hline C3/C4 L & 1.6 & 1.9 & 1.8 & 2.1 & 0.552 & 0.582 \\
\hline C4/C5 R & 1.7 & 2.0 & 2.1 & 2.1 & 0.548 & 0.689 \\
\hline C4/C5 L & 1.9 & 2.1 & 2.1 & 2.3 & 0.618 & 0.620 \\
\hline C5/C6 R & 2.4 & 2.6 & 2.5 & 2.5 & 0.614 & 0.721 \\
\hline C5/C6 L & 2.0 & 2.3 & 2.2 & 2.4 & 0.546 & 0.561 \\
\hline C6/C7 R & 1.9 & 2.1 & 2.1 & 2.1 & 0.662 & 0.661 \\
\hline C6/C7 L & 1.9 & 2.0 & 2.1 & 2.0 & 0.594 & 0.706 \\
\hline C7/T1 R & 1.1 & 1.1 & 1.1 & 1.2 & 0.582 & 0.262 \\
\hline C7/T1 L & 1.1 & 1.1 & 1.1 & 1.2 & 0.700 & 0.531 \\
\hline
\end{tabular}

1 , no stenosis -4 , severe stenosis. Interreader agreement evaluated by weighted $\kappa . R$ right, $L$ left of the 3D sequence (including reduced slice thickness with the potential of better visualization of the foramina even in a strictly sagittal plane). However, the differences between sequences were limited, visibility of foraminal contents and other structures being overall graded as "good" on both sequences with the exception of the foraminal vessel (the latter not representing the main structure of interest in foraminal imaging), and consequently do not appear to be significant for daily clinical practice.

\section{Foraminal stenosis grading}

Prior studies on the use of 3D T2w SPACE in the cervical spine mostly assessed the visibility of anatomical structures [13-15], mainly in smaller cohorts of healthy volunteers [13, 15] and in comparison to standard 2D T2w TSE. The present study also analyzed its use for a clinical indication, namely foraminal stenosis grading in a patient population, comparing it to a currently used dedicated sequence for foraminal analysis, sagittal oblique T2w TSE.

Stenosis grades were not significantly different between sequences at most levels, while higher grades were attributed at certain mid-cervical levels based on 3D T2w SPACE at 1.5 T. Underestimation and overestimation of stenosis on the 2D T2w TSE sequence seems possible due to its inherently greater slice thickness and spacing, with the potential of volume averaging. The single plane coverage of all neuroforamina of one side, while they may have different orientations, may contribute to difficulties in evaluation. It remains unclear which sequence more closely reflected true foraminal stenosis, as there was no reference standard for the evaluation. Prior studies on the grading of cervical foraminal stenosis focused mainly on the comparison of sagittal oblique $2 \mathrm{D} \mathrm{T} 2 \mathrm{w}$ sequences $[7,16]$ or $3 \mathrm{D} \mathrm{T} 2 \mathrm{w}$ sequences $[22,23]$ with conventional, i.e., sagittal and axial planes.
Stenosis prevalence and mean stenosis grades in the present study were low, ranging from 1.1 to 2.6. Additional investigations in cohorts with more prevalent significant foraminal stenosis as well as correlation with symptoms and surgical findings could clarify the respective sensitivity and specificity of 3D T2w and 2D T2w sequences.

\section{Acquisition time/time to complete grading}

Compared to the acquisition time of bilateral sagittal oblique 2D T2w TSE sequences, acquisition time of the 3D T2w SPACE sequence, which was accelerated by parallel imaging (iPAT of 2-3), was about $30 \%$ longer at $3 \mathrm{~T}(04: 31 \mathrm{~min}$ vs. 03:06 $\mathrm{min})$, and slightly shorter at 1.5 $\mathrm{T}$ (04:28 $\mathrm{min}$ vs. 04:54 $\mathrm{min})$, respectively. Within these acquisition times, 3D T2w SPACE also offers increased anatomical coverage in comparison to sagittal oblique 2D T2w TSE sequences, including the neuroforamina of both sides as well as the entire spinal canal, thereby offering an additional possibility to analyze structures in the spinal canal (particularly the nerve rootlets, better delineated than on $2 \mathrm{D} \mathrm{T} 2 \mathrm{w}$ according to prior studies [14]) and the potential to follow rootlets and proximal nerve roots on one single sequence. The possibility to reformat the sequence in arbitrary planes is of potential additional benefit, as the foramina of different levels may not lie in the same plane, particularly in patients with scoliosis or vertebral segmentation anomalies (Fig. 5). In this context, the longer time necessary for grading the foraminal stenosis compared to $2 \mathrm{D}$ T2 $\mathrm{w}$ TSE-less than $1 \mathrm{~min}$, including the time needed to reformat the images-appears acceptable for clinical practice.

In light of the relatively low additional examination and interpretation time and its added benefits, 3D T2w SPACE 

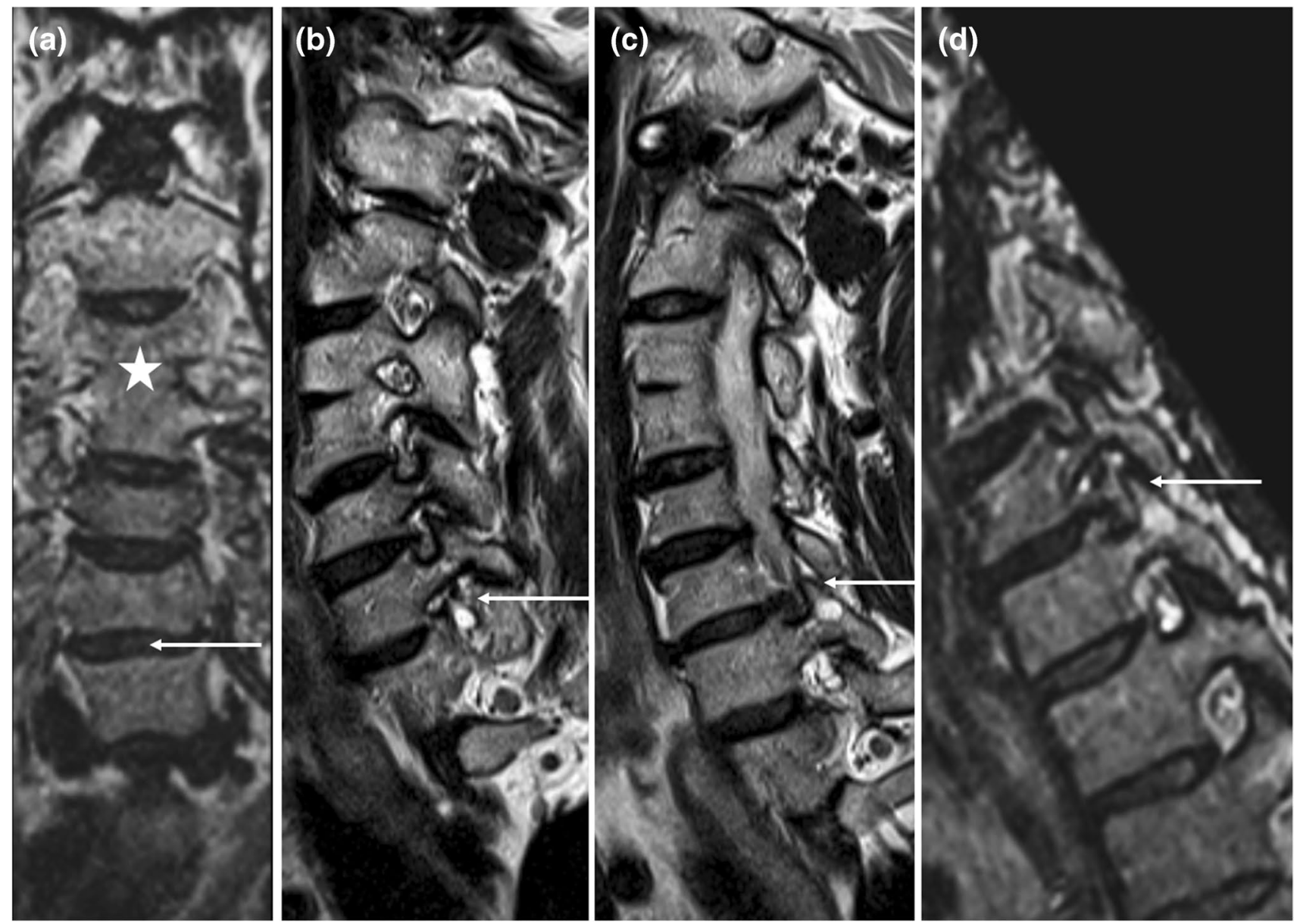

Fig. 5 a Coronal MPR of 3D T2w SPACE shows block vertebra C3/ $\mathrm{C} 4$ (asterisk) and slightly dextroconvex scoliotic posture centered on C6/C7 (arrow). b and c On sagittal oblique 2D T2w TSE (planned according to higher cervical segments) borders of the right $\mathrm{C} 6 / \mathrm{C} 7$ neuroforamen are on different slices (arrows), impairing the visu-

could supplant sagittal oblique 2D T2w TSE sequences. Replacement of conventional sagittal and axial T2w TSE and/or spoiled $\mathrm{T} 2 * \mathrm{w}$ sequences (such as multi-echo data image combination, MEDIC) seems less practicable unless for indications where the spinal cord itself is not of interest, as spinal cord anatomy and pathology are superiorly depicted on the latter sequence [24-28].

This study is limited by its retrospective design and the absence of a surgical reference standard. Further, the prevalence of significant foraminal stenosis of the cervical spine was low; however, this reflects the prevalence in a typical clinical setting.

3D T2w SPACE is comparable with sagittal oblique 2D T2w TSE in the evaluation of cervical neural foramina and can be acquired and analyzed in an acceptable time, furthering its potential to replace sagittal oblique sequences. alization of the neuroforamen and its contents. d Representation of the entire right $\mathrm{C} 6 / \mathrm{C} 7$ neuroforamen on a single slice on a sagittal oblique MPR of 3D T2w SPACE, allowing for easier appreciation of foraminal stenosis

Funding Open access funding provided by University of Zurich.

\section{Declarations}

Ethical approval Ethical approval from the local Ethics Committee was obtained for this retrospective study from data obtained for clinical purposes.

Informed consent Written informed consent was not required because subjects gave general consent to the institution.

Conflict of interest The authors declare that they have no conflict of interest.

Open Access This article is licensed under a Creative Commons Attribution 4.0 International License, which permits use, sharing, adaptation, distribution and reproduction in any medium or format, as long as you give appropriate credit to the original author(s) and the source, provide a link to the Creative Commons licence, and indicate if changes 
were made. The images or other third party material in this article are included in the article's Creative Commons licence, unless indicated otherwise in a credit line to the material. If material is not included in the article's Creative Commons licence and your intended use is not permitted by statutory regulation or exceeds the permitted use, you will need to obtain permission directly from the copyright holder. To view a copy of this licence, visit http://creativecommons.org/licenses/by/4.0/.

\section{References}

1. Freund M, Sartor K. Degenerative spine disorders in the context of clinical findings. Eur J Radiol. 2006;58(1):15-26.

2. Humphreys SC, An HS, Eck JC, Coppes M, Lim TH, Estkowski L. Oblique MRI as a useful adjunct in evaluation of cervical foraminal impingement. J Spinal Disord. 1998;11(4):295-9.

3. Simpson AK, Sabino J, Whang P, Emerson JW, Grauer JN. The assessment of cervical foramina with oblique radiographs: the effect of film angle on foraminal area. J Spinal Disord Tech. 2009;22(1):21-5.

4. Schell A, Rhee JM, Holbrook J, Lenehan E, Park KY. Assessing foraminal stenosis in the cervical spine: a comparison of threedimensional computed tomographic surface reconstruction to twodimensional modalities. Global Spine J. 2017;7(3):266-71.

5. Flannigan BD, Lufkin RB, McGlade C, Winter J, Batzdorf U, Wilson G, et al. MR imaging of the cervical spine: neurovascular anatomy. AJR Am J Roentgenol. 1987;148(4):785-90.

6. Modic MT, Masaryk TJ, Ross JS, Mulopulos GP, Bundschuh CV, Bohlman H. Cervical radiculopathy: value of oblique MR imaging. Radiology. 1987;163(1):227-31.

7. Shim JH, Park CK, Lee JH, Choi JW, Lee DC, Kim DH, et al. A comparison of angled sagittal MRI and conventional MRI in the diagnosis of herniated disc and stenosis in the cervical foramen. Eur Spine J. 2009;18(8):1109-16.

8. Kintzele L, Rehnitz C, Kauczor HU, Weber MA. Oblique sagittal images prevent underestimation of the neuroforaminal stenosis grade caused by disc herniation in cervical spine MRI. Rofo. 2018;190(10):946-54.

9. Schroeder GD, Suleiman LI, Chioffe MA, Mangan JJ, McKenzie JC, Kepler CK, et al. The effect of oblique magnetic resonance imaging on surgical decision making for patients undergoing an anterior cervical discectomy and fusion for cervical radiculopathy. Int J Spine Surg. 2019;13(3):302-7.

10. Wolansky LJ, Parikh DD, Shah KJ, Yalamanchili R, Farkas J. Magnetic resonance imaging protocols for cervical disc disease: what is your neighbor up to? J Neuroimaging. 2005;15(2):183-7.

11. Jung JY, Jee WH, Park MY, Lee SY, Kim JM. Meniscal tear configurations: categorization with 3D isotropic turbo spin-echo MRI compared with conventional MRI at $3 \mathrm{~T}$. AJR Am J Roentgenol. 2012;198(2):W173-180.

12 Lee S, Jee WH, Jung JY, Lee SY, Ryu KS, Ha KY. MRI of the lumbar spine: comparison of 3D isotropic turbo spin-echo SPACE sequence versus conventional $2 \mathrm{D}$ sequences at 3.0 T. Acta Radiol. 2015;56(2):174-81.

13. Meindl T, Wirth S, Weckbach S, Dietrich O, Reiser M, Schoenberg SO. Magnetic resonance imaging of the cervical spine: comparison of 2D T2-weighted turbo spin echo, 2D T2*weighted gradient-recalled echo and 3D T2-weighted variable flip-angle turbo spin echo sequences. Eur Radiol. 2009;19(3):713-21.
14. Chokshi FH, Sadigh G, Carpenter W, Allen JW. Diagnostic Quality of 3D T2-SPACE Compared with T2-FSE in the Evaluation of Cervical Spine MRI Anatomy. AJNR Am J Neuroradiol. 2017;38(4):846-50.

15. Kwon JW, Yoon YC, Choi SH. Three-dimensional isotropic T2-weighted cervical MRI at 3T: comparison with two-dimensional T2-weighted sequences. Clin Radiol. 2012;67(2):106-13.

16. Park MS, Moon SH, Lee HM, Kim TH, Oh JK, Lee SY, et al. Diagnostic value of oblique magnetic resonance images for evaluating cervical foraminal stenosis. Spine J. 2015;15(4):607-11.

17. Li T, Mirowitz SA. Comparative study of fast MR imaging: quantitative analysis on image quality and efficiency among various time frames and contrast behaviors. Magn Reson Imaging. 2002;20(6):471-8.

18. Park HJ, Kim SS, Lee SY, Park NH, Chung EC, Rho MH, et al. A practical MRI grading system for cervical foraminal stenosis based on oblique sagittal images. Br J Radiol. 2013;86(1025):20120515.

19. Harris PA, Taylor R, Minor BL, Elliott V, Fernandez M, O’Neal $\mathrm{L}$, et al. The REDCap consortium: building an international community of software platform partners. J Biomed Inform. 2019;95:103208.

20. Harris PA, Taylor R, Thielke R, Payne J, Gonzalez N, Conde JG. Research electronic data capture (REDCap)-a metadatadriven methodology and workflow process for providing translational research informatics support. J Biomed Inform. 2009;42(2):377-81.

21. Landis JR, Koch GG. The measurement of observer agreement for categorical data. Biometrics. 1977;33(1):159-74.

22. Kim W, Ahn KS, Kang CH, Kang WY, Yang KS. Comparison of MRI grading for cervical neural foraminal stenosis based on axial and oblique sagittal images: concordance and reliability study. Clin Imaging. 2017;43:165-9.

23. Fu MC, Buerba RA, Neway WE 3rd, Brown JE, Trivedi M, Lischuk AW, et al. Three-dimensional isotropic MRI of the cervical spine: a diagnostic comparison with conventional MRI. Clin Spine Surg. 2016;29(2):66-71.

24. Vertinsky AT, Krasnokutsky MV, Augustin M, Bammer R. Cutting-edge imaging of the spine. Neuroimaging Clin N Am. 2007; 17(1):117-36.

25. Held P, Seitz J, Frund R, Nitz W, Lenhart M, Geissler A. Comparison of two-dimensional gradient echo, turbo spin echo and two-dimensional turbo gradient spin echo sequences in MRI of the cervical spinal cord anatomy. Eur J Radiol. 2001;38(1):64-71.

26. Held P, Dorenbeck U, Seitz J, Frund R, Albrich H. MRI of the abnormal cervical spinal cord using 2D spoiled gradient echo multiecho sequence (MEDIC) with magnetization transfer saturation pulse. A T2* weighted feasibility study. J Neuroradiol. 2003;30(2):83-90.

27. Katz BH, Quencer RM, Hinks RS. Comparison of gradientrecalled-echo and $\mathrm{T} 2$-weighted spin-echo pulse sequences in intramedullary spinal lesions. AJNR Am J Neuroradiol. 1989;10(4):815-22.

28. Martin N, Malfair D, Zhao Y, Li D, Traboulsee A, Lang D, et al. Comparison of MERGE and axial T2-weighted fast spin-echo sequences for detection of multiple sclerosis lesions in the cervical spinal cord. AJR Am J Roentgenol. 2012;199(1):157-62.

Publisher's note Springer Nature remains neutral with regard to jurisdictional claims in published maps and institutional affiliations. 